\title{
Artículos
}

\section{El trabajo doméstico y de cuidado: su importancia y principales hallazgos en el caso mexicano}

\section{Domestic and care work: Their importance and main findings in the case of Mexico}

\author{
Brígida García Guzmán*
}

\begin{abstract}
Resumen
El objetivo de este artículo es analizar la importancia del trabajo doméstico y de cuidado para consumo final en las familias u hogares, así como examinar los principales hallazgos de la investigación sociodemográfica mexicana en torno al mismo. Inicialmente nos interesan los avances conceptuales y el reconocimiento de este tipo de trabajo en las estadísticas laborales y no laborales. Luego exploramos los resultados de distintas investigaciones respecto a aspectos tales como la calidad de la información recolectada sobre el trabajo doméstico y de cuidado, el valor económico de las actividades involucradas, la cantidad de tiempo invertido en ellas por diferentes grupos poblacionales, así como las relaciones que se establecen entre el desempeño del trabajo remunerado y el no remunerado. Finalmente, discutimos sobre el significado de los avances para la investigación y la acción pública futuras.
\end{abstract}

Palabras clave: trabajo no remunerado; trabajo doméstico; cuidado; sistemas de cuidado.

\begin{abstract}
The purpose of this article is to analyze the importance of domestic and care work for final consumption in families or households, and to examine the main findings of Mexican socio-demographic research on this issue. Initially, we are interested in the
\end{abstract}

Nota de la autora: Agradezco los comentarios de dos evaluadoras/es externos, quienes leyeron una primera versión de este texto con mucho interés y detenimiento, y aportaron valiosas sugerencias para mejorarlo.

* El Colegio de México, A.C., Centro de Estudios Demográficos, Urbanos y Ambientales. Dirección postal: Carretera Picacho-Ajusco 20, Ampliación Fuentes del Pedregal, Tlalpan, 14110, Ciudad de México, México. Correo electrónico: bgarcia@colmex.mx ORCID: https:// orcid.org/0000-0003-2223-5225 
conceptual advances and the acknowledgement of this type of work in labor and non-labor statistics. We subsequently explore the results of various studies on aspects such as the quality of the information collected on domestic work and care, the economic value of the activities involved, the amount of time spent on them by various population groups, as well as the relationships established between the performance of paid and unpaid work. Lastly, we discuss the meaning of the advances for future research and public action.

Keywords: unpaid work; domestic work; care; care systems.

\section{Introducción}

En la primera y segunda décadas del siglo XXI se han logrado adelantos importantes en el reconocimiento y la medición de la contribución económica y social de los trabajos no remunerados. Éstos pueden adquirir diferentes formas, como son la producción agrícola para el autoconsumo, el trabajo doméstico y de cuidado, el trabajo de aprendices en las empresas y el trabajo voluntario o comunitario. En este artículo nos interesa principalmente el trabajo doméstico y de cuidado para su consumo final en las familias u hogares. Nuestro objetivo es sistematizar y evaluar los avances logrados en diferentes aspectos sobre este tipo de trabajo, así como plantear los retos que enfrentamos en la investigación sociodemográfica en torno al mismo. No se pretende ofrecer un panorama exhaustivo, sino analizar las investigaciones que, desde nuestro punto de vista, han contribuido en mayor medida al desarrollo y consolidación de este campo de estudio en México.

Inicialmente nos interesa abordar el momento en que nos encontramos con respecto a la evolución conceptual. Aquí hacemos alusión al debate sobre el trabajo doméstico y su significado para el sistema capitalista y la reproducción social que tuvo lugar en los años sesenta y setenta del siglo $\mathrm{XX}$, así como al desplazamiento actual hacia la perspectiva del cuidado, $\mathrm{o}$ de la economía del cuidado, donde tiene un peso importante la contribución de este tipo de trabajo al bienestar individual y familiar. En esta primera sección también indicamos la importancia de las reflexiones en torno a la organización social del cuidado y su condición de derecho humano, y finalizamos con una discusión sobre la terminología utilizada a lo largo del artículo.

Enseguida centramos la atención en el reconocimiento de las actividades no remuneradas en las estadísticas laborales y no laborales. Aquí nos ocupamos de las últimas resoluciones de los estadísticos internacionales del trabajo, y de las controversias que ha suscitado la ampliación de la noción de trabajo. Detallamos además las principales fuentes de información con 
las que hoy se cuenta en México en lo que toca al trabajo doméstico y de cuidado. En una sección posterior, nos detenemos en los ejercicios de evaluación de la calidad de los datos recolectados y de valorización del trabajo no remunerado como parte de la contabilidad nacional.

Una vez examinados los antecedentes conceptuales y los aspectos que hemos considerado más centrales referentes a las estadísticas recolectadas en México, analizamos los hallazgos de investigación propiamente dichos y su relevancia. Dichos hallazgos cubren un número importante de aspectos, pero principalmente se centran en la estimación de la carga global de trabajo remunerado y no remunerado de mujeres y hombres pertenecientes a diferentes grupos poblacionales y familiares en el país. El esfuerzo está encaminado a precisar la magnitud de esa carga, generalmente desfavorable para las mujeres, en una sociedad desigual como la mexicana, desde numerosos puntos de vista: se consideran la situación de pobreza, la residencia ruralurbana, la condición indígena y no indígena, la pertenencia a distintos estratos de edad, composición, jefatura y ciclo vital familiar, para mencionar las dimensiones que más han sido tomadas en cuenta. Asimismo, analizamos los resultados de contribuciones más específicas que abordan la distribución e implicaciones del ejercicio del trabajo no remunerado en instancias particulares, tales como las parejas de doble ingreso, la población desempleada o la población adolescente. Finalmente, exploramos las investigaciones que detallan el efecto restrictivo del trabajo doméstico y de cuidado sobre la participación laboral femenina.

En el último apartado del artículo sintetizamos lo que consideramos son los principales aportes y limitaciones, tanto en lo que se refiere a la generación de información como a los resultados concretos de la investigación. Además, abordamos las principales carencias de nuestra organización social del cuidado y los principales desafíos que se presentan para la acción pública en el presente y futuro cercanos.

\section{Avances conceptuales en torno al trabajo doméstico y de cuidado}

Es frecuente que el trabajo remunerado, de carácter asalariado o no asalariado, sea el único que se reconozca como importante para garantizar el intercambio de bienes y servicios necesarios para la sobrevivencia humana. No obstante, existen muchos otros tipos de trabajo que no se remuneran y que son indispensables para reproducir las condiciones de vida y las relaciones sociales. Como bien aclaran Carrasco, Borderías y Torns (2011), el trabajo 
no remunerado ha estado siempre presente en muchas sociedades, pero apenas desde el siglo XX se comenzó a visibilizar y a constituir como objeto de estudio en algunas ciencias sociales como la historia, la sociología y la economía.

Muchos autores indican que el debate sobre el trabajo doméstico que tuvo inicialmente lugar en los años sesenta y setenta del siglo pasado, impulsado por el movimiento de las mujeres, contribuyó en gran medida a ampliar la reflexión conceptual en el tema que nos ocupa. La aportación del trabajo doméstico a la reproducción social y de la fuerza de trabajo, los planteamientos sobre la relación entre capitalismo y patriarcado, así como las iniciativas en torno al salario para el ama de casa, representaron aspectos centrales de este debate y sus propuestas, en el cual participaron científicos sociales y activistas de varias tendencias y nacionalidades (véase la recopilación de Rodríguez y Cooper, 2005, así como las contribuciones de Federici, 2013, y en México la investigación realizada por De Barbieri, 1984). Por un lado, se hacía hincapié en el subsidio que el trabajo doméstico hacía al sistema capitalista al contribuir a mantener el valor de la fuerza de trabajo por debajo del costo de su reproducción; se argumentaba que el salario pagado al trabajador no incluía el valor del trabajo doméstico y de los cuidados necesarios para su reproducción, de manera que esto contribuía a una mayor extracción de plusvalía y por lo tanto de acumulación de capital. Desde otra perspectiva, más bien se subrayaba lo esencial que era el trabajo doméstico para la reproducción social y, por lo tanto, para la sobrevivencia del modo capitalista de producción, que necesitaba de individuos libres que ofrecieran su fuerza de trabajo en el mercado (véase Rodríguez Enríquez, 2012).

En años más recientes, los avances conceptuales en torno a la comprensión del significado del trabajo no remunerado se han desplazado hacia la perspectiva del cuidado (o de los trabajos domésticos y de cuidados) como elementos indispensables que garantizan la reproducción social y el bienestar de las personas, pero que recaen principalmente sobre las mujeres. Nancy Folbre, una autora pionera y de mucha influencia en este campo, indica que con el concepto de cuidado es importante hacer referencia tanto a los servicios no pagados que se llevan a cabo en el interior de las familias, como a los servicios pagados en el mercado. Ha explorado las diferentes causas que llevan a la subvaloración del trabajo de cuidado en sus múltiples facetas y ha hecho hincapié en que el cuidado nunca se puede reducir a términos puramente económicos, pues conlleva aspectos emocionales. Son especialmente significativas sus reflexiones acerca del cuidado y su relación con las transferencias de recursos financieros y los conflictos de distribución que éste suscita entre diferentes sectores sociales; además, ha profundizado en 
las penalidades económicas de que son objeto las mujeres por la dedicación al trabajo de cuidado a lo largo de su ciclo de vida (véase Folbre, 2014).

En el contexto de los países desarrollados, pero no solamente allí, se han enfatizado mucho los aspectos afectivos e intersubjetivos del trabajo de cuidado, así como su diferenciación de otros tipos de actividades que tienen lugar en la esfera mercantil, y por lo tanto la necesidad de examinarlos con herramientas teóricas y metodológicas particulares (Carrasco, Borderías y Torns, 2011; Vega y Gutiérrez Rodríguez, 2014). Asimismo, hay quienes subrayan los aspectos morales y simbólicos de la ética del cuidado, en la cual estarían especialmente presentes los aspectos relacionales y contextuales (Arango Gaviria y Pascale Molinier, 2011). Es importante también señalar la vinculación de la perspectiva del cuidado con el análisis de las políticas públicas y sus deficiencias, en ámbitos nacionales y trasnacionales, especialmente en contextos como el europeo y el de algunos países de América Latina, donde están presentes el envejecimiento poblacional y/o la inmigración de personas que desempeñan servicios domésticos y de cuidado. En estas circunstancias, se hace cada vez más evidente la doble presencia, el segundo turno, la doble jornada de las mujeres que han incrementado su participación en los mercados laborales nacionales y en las transferencias o cadenas globales de cuidado. ${ }^{1}$

Para los fines específicos de este artículo, nos interesa detenernos en la contribución de un grupo de economistas feministas latinoamericanas, quienes se han empeñado en teorizar sobre la denominada "economía del cuidado", así como en especificar sus particularidades desde la perspectiva de la realidad de nuestra región. ${ }^{2}$ Algunas de estas estudiosas plantean la posibilidad de que las tareas domésticas y de cuidado constituyen el eslabón perdido del sistema económico, como lo ha planteado Corina Rodríguez Enríquez en el artículo antes mencionado. En esta corriente se hace hincapié en la relevancia de modificar el foco central del análisis en la disciplina económica, pasando de la elección de bienes y servicios que se intercambian en el mercado, hacia la provisión, es decir, al estudio de los bienes y procesos necesarios para la supervivencia humana (Rodríguez Enríquez, 2012; Esquivel, 2012a). Por lo

1 Exposiciones y análisis sobre el tránsito del debate del trabajo doméstico a la perspectiva del cuidado pueden ser encontrados en Carrasco, Borderías y Torns, 2011; Esquivel, 2012a; Rodríguez Enríquez, 2012; Ceballos, 2013; Aguirre y Ferrari, 2014; Vega y Gutiérrez Rodríguez, 2014. La dimensión trasnacional o global actual de los trabajos domésticos y de cuidado y sus distintas facetas, tanto en términos conceptuales como en la investigación sociodemográfica, ha sido desarrollada por autoras como Yeates, 2005; Cerrutti, Maguid y Binstock, 2013; Ariza, 2014.

2 Un acucioso y detallado relato del surgimiento y desarrollo de la economía feminista, así como de las diferencias y matices entre autoras italianas, francesas y anglosajonas, puede ser encontrado en Carrasco, Borderías y Torns, 2011. 
anterior, no sólo se postula la necesidad de analizar de manera conjunta el proceso de producción material y el de reproducción de las personas, sino que se centra la atención en el bienestar social derivado del trabajo doméstico y de cuidado. Esto es, para el caso de países como México, consideramos relevante el planteamiento de que el trabajo reproductivo no remunerado cubre el desfase entre los recursos disponibles y los consumos familiares efectivos, es decir, entre las condiciones imperantes en el mercado de trabajo y las condiciones de vida (véase Rodríguez Enríquez, 2012; Esquivel, 2012b).

En lo que toca a la perspectiva del cuidado en conexión con el análisis de los regímenes de bienestar, hay varias aristas que nos importa considerar. En el plano más abstracto, se plantea que las familias deben de ser vistas como proveedoras de bienestar y que cada régimen de bienestar descansa en un régimen particular de cuidado. Lo importante a subrayar sería entonces la repartición del cuidado entre las familias, la comunidad, el mercado y el Estado. ${ }^{3}$ Asimismo, habría que preguntarse principalmente sobre la demanda de cuidados, indagar acerca del perfil de las personas que cuidan, así como sobre la distribución del cuidado y su retribución, aspectos de especial relevancia en el análisis y diseño de las políticas sociales y en lo que se ha dado en llamar la organización social del cuidado. En este entorno, se apunta que la perspectiva del cuidado permite articular políticas sectoriales, y que ésta ha cobrado especial vigencia ante el aumento del trabajo extradoméstico femenino y las demandas de ciudadanía social de las mujeres (véase Arriagada y Todaro, 2012; Aguirre y Ferrari, 2014; Batthyány, 2015).

Otro ángulo del cuidado que ha despertado interés en América Latina es su condición de derecho humano fundamental: derecho a cuidar y a ser cuidado. Quienes enfatizan esta dimensión también se preocupan por clarificar que el Estado no sólo debería compensar las prestaciones que no se obtienen en el mercado, sino que debería ser garante del cuidado como derecho universal de todas las personas. Si el Estado sólo se limita a ofrecer servicios que alivien la situación de las mujeres y de los más vulnerables, lo que se refuerza es la división del trabajo predominante (véase Montaño y Calderón, 2010; Batthyány, 2015).

Para finalizar este apartado, queremos reflexionar sobre la utilización de los términos en el campo de los estudios sobre el trabajo no remunerado,

3 En estos planteamientos se aprecia la influencia del importante teórico de los regímenes de bienestar, Gosta Esping-Andersen (véase Martínez Franzoni y Koen Voorend, 2013, para la región latinoamericana). La perspectiva del cuidado en el ámbito de las políticas públicas ha sido impulsada de manera especial por los diferentes órganos del Sistema de Naciones Unidas, y ha sido un foco central de las diferentes conferencias mundiales y latinoamericanas sobre las mujeres (para un recuento al respecto, véase Aguirre y Ferrari, 2014). 
y de manera más específica en lo que corresponde a los trabajos domésticos y de cuidado. De inicio hay que mencionar que muchos especialistas de diversas nacionalidades han adoptado la perspectiva y el término del cuidado (o de los cuidados directos e indirectos) para referirse tanto al cuidado de personas de diferentes edades y condición de dependencia, como a las tareas domésticas (alimentación, limpieza de los hogares y viviendas, gestión de la vida cotidiana). Suponemos que esto ha ocurrido porque implícitamente consideran que todas estas actividades, directa o indirectamente, estarían finalmente encaminadas a proveer bienestar y a garantizar la subsistencia humana. Otros estudiosos, especialmente en los países desarrollados, se adhieren a la perspectiva del cuidado para referirse de manera exclusiva al cuidado de personas dependientes, y se adhieren a este enfoque y reivindican el uso del término para referirse a las tareas que implican una perspectiva relacional, difícil de reemplazar y de sustituir en el mercado (véase el recuento de Esquivel, 2012a, y también las reflexiones sobre las distintas dimensiones presentes en el ejercicio del cuidado en Carrasco, Borderías y Torns, 2011; Arango Gaviria y Molinier, 2011a; Martín Palomo, 2011; Pautassi y Zibecchi, 2013; Vega y Gutiérrez Rodríguez, 2014).

A diferencia de lo anterior, hay quienes, especialmente desde el pensamiento latinoamericano, reivindican la importancia y la necesidad de analizar por separado al trabajo doméstico y al de cuidado. Desde nuestra óptica, la posición de Esquivel (2012a) es la más convincente en esta dirección, pues esta autora hace hincapié en que cada uno de estos trabajos tiene sus particularidades, actores e instituciones relacionadas en sociedades desiguales y pobres como las nuestras. Esquivel (2012a) introduce en el debate aspectos tales como los distintos significados de los términos en sociedades desarrolladas y en desarrollo, los diversos niveles de análisis involucrados, así como la posibilidad de que haya agendas políticas también diferenciadas; argumenta además que en América Latina puede no haber diferentes grados de mercantilización entre el trabajo doméstico y de cuidado, como se apunta en el caso de algunos países desarrollados. ${ }^{4}$

Ahora bien, hay que subrayar también que los referentes simbólicos para el trabajo doméstico y de cuidados pueden ser distintos, lo cual es relevante para un caso como el mexicano y el de países similares. Teresa Torns analiza este último aspecto y plantea que el término cuidado puede ser más fácil de reivindicar en términos positivos que el de trabajo doméstico, pues éste último muchas veces se liga al mundo de los sirvientes (Torns, 2008). Por

4 Es decir, tanto el trabajo doméstico como el de cuidado pueden ser objeto de transacciones mercantiles en el caso de algunos sectores sociales. 
todo lo anterior, en este texto -y en otros que hemos escrito- nos hemos acogido a la idea de distinguir entre el estudio del trabajo doméstico y el del cuidado, así como considerar por separado la naturaleza de ambos y los diversos aspectos relacionados.

\section{Reconocimiento del trabajo no remunerado en las fuentes de información estadística}

El reconocimiento de los trabajos no remunerados en las estadísticas nacionales e internacionales, así como la ampliación de fuentes de información al respecto, ha sido considerable en los últimos años. Varios son los aspectos que podemos puntualizar en este campo, el cual ha sido objeto de discusiones frecuentes en todas las conferencias sobre las mujeres organizadas por el sistema de Naciones Unidas, además de aquellas dedicadas al ámbito laboral propiamente dicho.

Habría que comenzar por indicar que en la resolución más relevante emanada de la última conferencia de estadísticos del trabajo (la XIX CIET de 2013), se amplía la noción de trabajo para incluir a la producción de bienes o prestación de servicios para el autoconsumo o uso final propio en las familias u hogares, incluido aquí el trabajo doméstico y de cuidado, entre otros tipos de trabajo (véase OIT, 2013; Negrete Prieto y Ramírez Reynoso, 2015). Dicha resolución es bastante amplia y compleja, pero nos interesa recalcar la diferencia que se ha establecido en este ámbito del autoconsumo. Hasta ahora, el único trabajo de autoconsumo que contaba con reconocimiento en las estadísticas laborales, y también en el Sistema de Cuentas Nacionales (frontera de producción), era la producción de bienes agrícolas de subsistencia y actividades relacionadas. Ahora también los servicios no remunerados para uso final en las familias u hogares, entre los que se incluyen los domésticos y de cuidado, cuentan con este reconocimiento formal.

Sin embargo, no hay que olvidar que en la XIX CIET también se definió de manera más restrictiva la noción de fuerza de trabajo. Ahora se propone que sólo aquellas personas que desempeñen un trabajo en una ocupación por un ingreso o beneficio formarían parte de la fuerza de trabajo. Y, por el contrario, todas las personas que desempeñen actividades de autoconsumo (ya sea de producción de bienes como en la agricultura de subsistencia, o de prestación de servicios como en los quehaceres del hogar) quedarían fuera de la fuerza de trabajo. ${ }^{5}$ En resumen, contamos en el momento actual con

5 En términos cuantitativos esta restricción afectaría principalmente a los trabajadores 
un reconocimiento de la Organización Internacional del Trabajo de todos los tipos de trabajo (incluyendo el doméstico y de cuidado), pero con una definición más estrecha de fuerza de trabajo. Hay que subrayar que la definición de fuerza de trabajo (o población económicamente activa) es crucial, no sólo en términos de medición y elaboración de indicadores laborales, económicos y de niveles de pobreza. Es usual que este subconjunto constituya una referencia en la identificación de sujetos susceptibles de ser beneficiados por las políticas públicas. Se trata pues de avances restringidos, controversiales y todavía sujetos a mucha discusión antes de su posible puesta en práctica; no obstante, son importantes desde la perspectiva de este artículo porque recogen parcialmente las sugerencias plasmadas en un sinnúmero de reuniones y documentos por los movimientos de mujeres -así como por la perspectiva de género en las ciencias sociales- de ampliar la noción de trabajo para visibilizar y valorar las actividades domésticas y de cuidado, entre otros aspectos.

Aunado al creciente reconocimiento del trabajo doméstico y de cuidado en los diversos tipos de estadísticas laborales, hay que hacer hincapié en la multiplicación de fuentes de información en los últimos lustros que permiten medir estos trabajos. A veces se trata de la inclusión de algunas preguntas en las encuestas de empleo, y a veces de fuentes específicas como son las encuestas de uso del tiempo, las cuales se sabe que son una fuente más idónea para la captación del trabajo no remunerado precisamente porque el tiempo invertido en estas actividades es el criterio principal para estimar su valor y contribución a la reproducción social (véase Durán, 2012; Aguirre y Ferrari, 2014).

En México se han levantado encuestas nacionales de uso del tiempo por parte del Instituto Nacional de Estadística y Geografía (INEGI) en 1996, 1998, 2002, 2009 y 2014; éstas constituyen instrumentos específicos mediante los cuales es posible profundizar en las particularidades del trabajo doméstico y de cuidado, entre otros aspectos. Asimismo, en 2012 se llevó a cabo la Encuesta Laboral y de Corresponsabilidad Social (ELCOS), que ofrece información para relacionar las condiciones femeninas en el mercado laboral y las tareas de cuidado que realizan en sus hogares (véase García y Pacheco, 2014; Pacheco, 2013). Además de lo anterior, en las encuestas nacionales de empleo se viene recolectando de tiempo atrás información sobre horas de trabajo doméstico y de cuidado. En la serie actual de la En-

en la agricultura de subsistencia, quienes hasta ahora en México formaban parte de la fuerza de trabajo o población económicamente activa. Por su parte, aquellas trabajadoras/es involucrados en los quehaceres del hogar nunca han sido considerados como parte de la población económicamente activa o de la fuerza de trabajo. 
cuesta Nacional de Ocupación y Empleo (ENOE) se incluyen preguntas sobre quehaceres domésticos, construcción, reparación y mantenimiento de la vivienda, realización de compras o trámites, transporte de los miembros del hogar, cuidado o atención sin pago a niños, ancianos, enfermos y discapacitados. Habría que mencionar también la recolección de datos sobre trabajo doméstico y de cuidado en la Encuesta Nacional de Ingresos y Gastos de los Hogares (ENIGH), en la cual se recolecta información sobre cuidado de niños, ancianos y enfermos, tareas de acarreo de agua, reparación y mantenimiento de la vivienda y los quehaceres del hogar. Un aspecto importante de la información sobre trabajo doméstico y de cuidado recolectada en las series ENOE y ENIGH, así como en encuestas sobre salud y seguridad social, y en la Encuesta Intercensal de 2015, es que permite relacionar elementos de las actividades no remuneradas con todas las baterías de información que usualmente se recolectan sobre ocupación y desocupación, salud y seguridad social, ingresos y consumo en los hogares mexicanos, entre otras muchas temáticas (véase Márquez Scotti y Mora Salas, 2014; Sánchez, 2014; Galindo, García y Rivera, 2015; Max, 2016).

\section{Evaluación de la información recolectada y cálculos sobre el valor económico del trabajo no remunerado}

\section{La calidad de los datos recolectados}

La discusión sobre cuál es el mejor instrumento para recolectar información sobre el tiempo dedicado a las tareas domésticas y de cuidado ya ha dado lugar a reflexiones muy nutridas en este campo de investigación. Un aspecto específico que motiva mucho debate se refiere a las posibles sobrestimaciones (o subestimaciones) en que se incurre con el levantamiento de un diario de actividades, en comparación con un cuestionario previamente estructurado por los generadores de información (véase Gandini y Parker, 2013; Damián, 2014a). Existe casi consenso al considerar que el diario de actividades es el instrumento más idóneo, pero se trata de un recurso muy oneroso y más fácilmente aplicable en sociedades donde el nivel de escolaridad de la población es relativamente elevado.

Damián (2014a) considera que el cuestionario estructurado que se ha utilizado en la mayor parte de las encuestas de uso del tiempo en el país ha llevado a sobrestimar, especialmente en el caso de las mujeres, el tiempo dedicado a las tareas domésticas y de cuidado, pues éste se calcula como una suma del tiempo empleado en un listado de actividades, el cual se ha 
ampliado de un levantamiento a otro. Otras investigaciones, como la llevada a cabo por Gandini y Parker (2013), llegan a conclusiones un tanto distintas, especialmente en lo que respecta a las brechas de género en el registro del uso del tiempo cuando se utilizan los diferentes tipos de instrumentos. Estas autoras llevaron a cabo una investigación para el caso de la Ciudad de México en la que compararon las respuestas obtenidas por la misma población masculina y femenina con un diario de actividades en la semana posterior al levantamiento en comparación con un cuestionario estructurado. Este estudio llega a la conclusión de que en el cuestionario estructurado lo que se tiende a sobrestimar son las actividades socialmente normadas, con horarios establecidos o esperados (trabajo remunerado, trabajo doméstico, cuidado de personas en el hogar, trabajo voluntario y el estudio). En cambio, los registros son menores en actividades asociadas a mayores procesos de individuación (actividades de convivencia y recreación, uso de medios masivos de comunicación y cuidados personales, incluyendo las horas de sueño). Siguiendo los razonamientos anteriores, se reporta que son los hombres los que sobrestiman su participación en el trabajo doméstico, y así se estima una brecha de género menor cuando se utiliza un cuestionario estructurado, que la que se obtiene con el uso de un diario de actividades. De cualquier manera, se comprueba además que los tiempos estimados con diferentes instrumentos de captación pueden ser diferentes, pero no así las tendencias que se obtienen con respecto a la edad, la condición de hombre o mujer y el estado conyugal.

Un punto de discusión frecuente se refiere a la inclusión de las actividades de "estar al pendiente" en lo que respecta al trabajo doméstico y de cuidado. Muchos autores no consideran dichas actividades en sus estimaciones de las cargas globales de trabajo porque elevan de manera apreciable dichas estimaciones. No obstante, otros argumentan que "estar al pendiente" implica un costo de libertad, y por lo tanto toman en cuenta una parte del tiempo declarado a dichas tareas. ${ }^{6}$ Estos debates y resultados nos motivan a seguir analizando la calidad de los datos que se generan en nuestro campo, ya que se trata de experiencias novedosas, tanto para los generadores de información como para la población entrevistada.

6 Véase la argumentación en Durán, 2012 y Pedrero, 2014, así como las correcciones a los datos de los autores que conforman el libro de García y Pacheco, 2014 y que están sintetizadas en la introducción del mismo. 


\section{Valor económico del trabajo doméstico y de cuidado}

Como apunta Pedrero (2014), es importante contar con una valoración económica del trabajo no remunerado porque mediante este esfuerzo se contribuye a la visibilización de dicho trabajo en una sociedad donde los factores económicos tienen un papel primordial. Según Durán (2012), la mayoría de los esfuerzos en esta dirección utilizan el método que denomina input, o sea la asignación de un costo de sustitución o de un costo de oportunidad de realizar el trabajo no remunerado, teniendo en cuenta la condición de hombre o mujer, la edad y el nivel educativo de quien lo desempeña. El costo del trabajo doméstico y de cuidado puede asimilarse al costo medio de la hora trabajada por el conjunto de los trabajadores o el que prevalece en los sectores más afines a estos trabajos.

Mercedes Pedrero y Teresa Rendón han sido pioneras en la estimación del valor económico del trabajo no remunerado en México. Pedrero ha utilizado tres métodos diferentes para esta cuantificación: el primero es la estimación del precio de mercado de los bienes y servicios derivados de la producción doméstica; el segundo se basa en la escolaridad de las amas de casa y los ingresos percibidos por las trabajadoras remuneradas conforme a su escolaridad; y el tercero descansa en el tiempo que se destina a las distintas actividades sin remuneración en el interior del hogar, a las cuales se les aplica el pago por hora de una actividad equivalente en el mercado (por ejemplo, para las actividades relacionadas con la educación, se puede tomar como referencia el sueldo medio de profesores en distintos niveles; para las actividades domésticas, el salario de diversos tipos de empleadas domésticas) (véase Pedrero, 2014 y Rendón, 2008).

Un elemento particularmente importante en este ámbito de interés es la estimación de cuentas satélite de trabajo no remunerado. Durán (2012) subraya que el Sistema de Cuentas Nacionales ( $\mathrm{SCN}$ ) abarca principalmente a las actividades de mercado, y que para no alterarlo se ha ideado el cálculo de estas cuentas satélite en diferentes rubros, las cuales se ubican fuera del cuerpo principal del sistema, pero utilizan un lenguaje afín para que el entendimiento sea posible. Lo que se busca es encontrar una unidad de cuenta común entre sistemas económicos que son heterogéneos, o al menos un sistema de conversión entre uno y otro. En el caso mexicano, el tiempo dedicado a las actividades no remuneradas y el pago por hora de una actividad similar en el mercado son criterios comunes en la estimación de las cuentas satélite. $^{7}$

7 Pedrero (2014) indica que lo que existe en México son estimaciones del valor econó- 
Existen ejercicios sobre cuentas satélite de trabajo no remunerado desde la década de los noventa del siglo pasado en los cuales se ha empleado la información proveniente de las ENUT y de las encuestas de empleo. Según la última estimación del INEGI de la cuenta satélite de trabajo no remunerado en 2013, el valor económico de las actividades domésticas y de cuidado equivale a $20.5 \%$ del producto interno bruto; se trata de un dato mayor al de cualquier sector económico tomado en cuenta de manera individual. Otras estimaciones del mismo valor basadas en la ENUT de 2009 arrojan cifras entre 21.7 y $22.6 \%$ del PIB (véase Pedrero, 2014). ${ }^{8}$

\section{Principales resultados de investigación 9}

Desde los años 1970 se llevaron a cabo en el país investigaciones pioneras sobre el trabajo doméstico principalmente desde un enfoque conceptual y/o cualitativo (véase Pedrero, 1977; De Barbieri, 1984; Sánchez Gómez y Martini Escobar, 1987; Torres, 1989; Goldsmith, 2007). Además, ya existen para la década de 1990 estimaciones innovadoras sobre la sobrecarga de trabajo femenino, con base en los datos que proporcionaban en ese entonces las encuestas de empleo (Oliveira, Ariza y Eternod, 1996). Por su parte, la información recolectada en la primera encuesta mexicana de uso del tiempo de 1996 fue aprovechada de manera exhaustiva por Rendón (2008), quien dio cuenta pormenorizada de la división del trabajo entre hombres y mujeres en las áreas rurales y urbanas del país. Una de sus principales conclusiones fue que la reproducción material de nuestra sociedad descansaba, todavía en esos años, en una vasta producción de bienes y servicios generados en los hogares.

Ahora bien, la investigación social en el campo del trabajo no remunerado en México se ha ampliado de manera importante en los dos primeros

mico del trabajo no remunerado y de la proporción que éste representa dentro del producto interno bruto (PIB). Según esta autora, faltarían otros elementos para el cálculo de una cuenta satélite de trabajo no remunerado, como serían la estimación del consumo de capital fijo y de los consumos intermedios de los hogares.

8 De manera más reciente también se ha estimado el valor económico del trabajo no remunerado destinado a los cuidados a la salud de la población adulta mayor (véase López Ortega, Licea, Figueroa y García, 2016). Estos autores concluyen que el trabajo no remunerado en salud en México es sustancial y similar a lo que representa la atención hospitalaria con cifras cercanas al 20\% del total del PIB en salud.

9 Esta revisión está hecha desde una perspectiva sociodemográfica y está centrada en los análisis empíricos publicados en México o sobre el país, durante las dos últimas décadas. Más que una revisión sistemática, lo que nos interesa en este apartado es dar cuenta de aquellos resultados que más aportan al conocimiento existente, desde nuestra experiencia de investigación en este tema. 
decenios del siglo XXI, en alguna medida debido a la ampliación de la información existente al respecto. Como hemos visto, algunos estudios se han abocado a evaluar la calidad de los datos recolectados, y otros han aportado al conocimiento en lo que respecta al valor económico de las actividades domésticas y de cuidado. Además de lo anterior, la investigación actual ha dado cuenta del ejercicio del trabajo no remunerado y de la carga global de trabajo (no remunerada y remunerada) entre mujeres y hombres de distintos grupos poblacionales y que enfrentan circunstancias familiares e individuales diferenciadas, como veremos en las páginas que siguen. Finalmente, han sido objeto de especial atención las limitaciones que se le presentan a la mayoría de las mujeres que son responsables del trabajo no remunerado para ejercer distintas opciones en el mercado de trabajo remunerado.

La última ENUT (2014) nos indica que las mujeres dedican al trabajo no remunerado de los hogares un promedio de 50.1 horas a la semana, en comparación con 17.6 horas por parte de los varones. En cambio, los varones dedican un promedio de 52.4 horas a la semana a las actividades para el mercado y bienes de autoconsumo, y las mujeres 33 horas. Si se suma el trabajo no remunerado y el remunerado, tenemos que la carga global de trabajo de las mujeres excede a la de los varones en alrededor de 13 horas semanales en promedio. Dicha cifra es similar a la obtenida con la ENUT de 2009, sin contar las actividades de "estar al pendiente" en el cuidado de las personas. México usualmente ocupa los primeros lugares en América Latina en lo que respecta a las horas dedicadas al trabajo doméstico y de cuidado, pero habría que recordar que estas estadísticas internacionales en nuestra región no parten de instrumentos homogéneos de captación de información (ENUT, 2014; Rodríguez y García, 2014; Arriagada, 2017). ${ }^{10}$

Aunque los varones mexicanos siguen la tendencia más conocida de un reducido involucramiento en la esfera reproductiva, es importante introducir matices y señalar diferencias entre ellos. Como suele ocurrir en otros contextos, los varones se involucran en mayor medida en el cuidado que en el trabajo doméstico propiamente dicho (limpieza, preparación de alimentos). Los datos de la ENUT (2014) indican que los hombres dedican 9.7 horas semanales en promedio a las actividades domésticas, 12.4 horas al cuidado y 14.8 horas a los cuidados especiales por enfermedad o discapacidad de los integrantes de los hogares. ${ }^{11}$

10 Debido a la falta de homogeneidad en los distintos instrumentos, es especialmente acertado que el indicador sobre trabajo no remunerado en los ODM (Objetivos de Desarrollo del Milenio) esté expresado en términos relativos, esto es, el porcentaje de tiempo dedicado a los quehaceres domésticos y de cuidados no remunerados por sexo, grupo de edad y ubicación.

11 Estos promedios son independientes entre sí porque se calculan para la población 
También es notoria la participación masculina en la reparación de las viviendas y en la producción de bienes de autoconsumo en las áreas rurales. Los más jóvenes (menores de 40 años) y los más educados (que cuentan con bachillerato y niveles superiores de escolaridad) reportan mayor cantidad de horas dedicadas al trabajo doméstico y de cuidado. Asimismo, la ocupación de la cónyuge, en el caso de los varones unidos o casados, es de vital importancia sobre todo si es asalariada (véase Rendón, 2008; Granados, 2013; Rodríguez y García, 2014; Rojas y Martínez, 2014; Galindo, García y Rivera, 2015).

Es en la etapa de formación familiar, o bien cuando las familias se encuentran en la etapa final, cuando se requiere de mayor cantidad de trabajo no remunerado y específicamente de las actividades relacionadas con el cuidado de niños y niñas pequeñas o de personas adultas mayores. Estos hallazgos cobran especial relevancia cuando recordamos que la mayoría de las mujeres mexicanas se encuentran precisamente en etapas de expansión familiar, que son también las de mayor demanda de trabajo remunerado (Nava, 2013; Rivero y Hernández, 2014). Como contraparte, también es interesante tener en cuenta que ha sido constatado que hay patrones de uso del tiempo con mayores espacios para el ocio y el descanso, así como para la ayuda a otros hogares y tareas comunitarias, especialmente en las edades adultas mayores (Rivero y Hernández, 2014).

En una sociedad tan desigual como la mexicana en términos socioeconómicos, es frecuente que interese documentar la cantidad de trabajo no remunerado y remunerado que llevan a cabo los grupos más desfavorecidos en comparación con los mejor ubicados. Se ha comprobado que en los hogares más pobres la carga de trabajo es mayor al compararla con otros estratos de ingreso, sobre todo en las áreas rurales (Inmujeres, 2005). Asimismo, Damián (2014b), desde la perspectiva de la pobreza de tiempo, ha encontrado, con base en las diversas encuestas, que existe un tercio de hogares consistentemente pobres, pues su ingreso es bajo y no tienen tiempo adicional a su disposición para dedicarlo al trabajo remunerado.

Las diferencias para las áreas rurales y urbanas se han hecho evidentes mediante el cálculo de tasas de participación en ambos contextos, y también por medio de la estimación de tiempos medios por participante y de tiempos medios sociales donde se estandarizan dichos tiempos medios por el nivel

específica que declara participar en cada actividad y las personas pueden involucrarse en más de una de estas tareas. Asimismo, la participación masculina puede ser diferencial en las distintas tareas de cuidado. Granados (2013) y también Jácome (2013) reportan, con base en la ELCOS 2012, mayor participación de los varones en el cuidado a enfermos temporales que en el de niños menores de 14 años. Por su parte, Rodríguez y García (2017) indican, con base en la Encuesta Intercensal de 2015, que las brechas de género son menores en el cuidado a adultos mayores que en el de menores de 14 años. 
de participación. En las áreas rurales sobresale además que, tanto en el caso de los hombres como de las mujeres, está presente en mayor medida el trabajo voluntario y de respaldo a otros hogares. De esta manera queda comprobada, mediante los datos de las encuestas de uso del tiempo, la tradición de solidaridad que muchas veces ha caracterizado a la población rural del país (véase Pacheco y Florez, 2014).

Un grupo que reviste una importancia particular por la vulnerabilidad de sus condiciones de vida es la población indígena. Se ha demostrado la necesidad de tener en cuenta una serie de factores intervinientes para poder llegar a conclusiones relevantes, como serían las condiciones de las viviendas, las características sociodemográficas, el nivel educativo, la condición de actividad económica, el acceso a servicios de salud, la afiliación a programas de apoyo gubernamentales y las necesidades de cuidado en sus hogares. Teniendo éstos en cuenta, se llega a la conclusión de que todos los miembros de los hogares indígenas tienen un involucramiento más acentuado (que los no indígenas) en las labores domésticas, de cuidado y en el trabajo voluntario (Jácome y Mier y Terán, 2014).

Los aspectos anteriores se refieren a los rasgos más generales del desempeño del trabajo doméstico y de cuidado, pero interesa destacar que tanto la información de las ENUT como la de la ELCOS y otras encuestas han permitido profundizar en otras facetas más específicas del ejercicio de dicho trabajo, así como de la carga global de trabajo remunerada y no remunerada. Desde esta perspectiva, hoy se conoce más sobre la distribución de los distintos tipos de trabajo en hogares con estructuras y/o etapas del ciclo vital familiar diferenciadas (se toma en cuenta la edad de los diferentes miembros y/o la presencia de parientes o no parientes, además de los jefes/jefas, esposas e hijos e hijas) (Nava, 2013; Orozco, 2014 y 2017; Santoyo y Pacheco, 2014). Asimismo, se ha profundizado en lo que sucede con la distribución del trabajo en las parejas de doble ingreso, donde se encuentra que el número de horas invertidas en el trabajo doméstico son más reducidas y la brecha de género es menor (Sánchez, 2014). También habría que mencionar los trabajos que se centran en la población desempleada y que analizan cómo los patrones de uso del tiempo y el desempeño del trabajo doméstico reducen las posibilidades de inserción laboral, especialmente para el caso femenino (Márquez y Mora, 2014; Márquez, 2017). Y sobresale además el estudio de la población adolescente y el tema de las niñas que cuidan niños (poco más de la mitad de las niñas y adolescentes urbanas de 14 a 17 años asume parte del cuidado de las nuevas generaciones) (Miranda, 2013).

Otros aspectos particulares examinados son los relacionados con las decisiones que toman las mujeres que cuidan y que no cuidan con respecto 
a dimensiones económicas, familiares y sociales que pueden vincularse con su mayor o menor autonomía (Florez, Pacheco y Pedrero, 2013). Han llamado adicionalmente la atención las expectativas laborales de las mujeres que no son económicamente activas y la exploración de las condiciones en las que estarían dispuestas a asumir la doble jornada de trabajo (Hernández, 2013). Y también se ha analizado la intensidad con la que se desempeña el trabajo de cuidado y las redes familiares y sociales involucradas (parentesco y otras características de las personas cuidadoras y de las que se cuidan; diversidad de las actividades que se llevan a cabo; y lugar donde se desarrollan las distintas tareas, ya sea en los hogares propios o en otros hogares) (Ceballos, 2013).

Finalmente, son de llamar la atención las investigaciones en torno al efecto restrictivo del trabajo doméstico y de cuidado sobre aspectos específicos de la participación laboral femenina. La información ahora existente les ha permitido a autoras como Karina Orozco calcular de manera directa las cargas domésticas y extradomésticas en los diversos arreglos y posiciones familiares, en vez de suponer su magnitud de manera indirecta -como en estudios previos-mediante variables como el número y la edad de los hijos. Las cargas domésticas estimadas directamente no sólo restringen la participación económica de las mujeres, sino que juegan un papel muy importante en la explicación de la brecha salarial existente entre hombres y mujeres asalariados, teniendo en cuenta una serie muy amplia de aspectos intervinientes (Orozco, 2014). Además, la situación ocupacional es muy importante, pues se han obtenido resultados distintos para las mujeres asalariadas y las que laboran por cuenta propia (Orozco, 2013).

\section{Discusión y consideraciones finales}

Los avances obtenidos de forma reciente en los aspectos conceptuales, en la generación de información y en la investigación concreta en torno al trabajo no remunerado en México y América Latina son sin duda significativos. Como hemos visto, se trata de un tema que había permanecido poco visible, tanto en los estudios de corte académico como en la política social. En el momento actual todo lo relacionado con el área de los cuidados ha recibido un impulso especial, sin duda relacionado con la necesidad de prever y proveer los servicios necesarios para la creciente población adulta mayor en México y en el mundo. En este contexto, resulta necesario iniciar esta discusión final con una nota de alerta para no dejar rezagado el estudio del trabajo doméstico, o para no subsumirlo bajo la acepción de un cuidado 
indirecto. Vimos antes que se trata de trabajos con actores, instituciones y relaciones sociales distintos en el caso de una sociedad menos desarrollada y muy desigual como la mexicana. Coincidimos con el planteamiento de que el trabajo de cuidado es más fácil de reivindicar en términos positivos, y es significativo que algunos varones se estén comenzando a involucrar en algunas facetas del mismo. En cambio, el trabajo doméstico sigue teniendo en México connotaciones de servidumbre y no tenemos señales de mayor desempeño masculino en estas tareas. Tanto en lo que respecta al mercado de trabajo como en las explicaciones de la subordinación femenina, consideramos relevante mantener por separado lo relacionado con el trabajo doméstico y el de cuidados. En términos de política pública, las reflexiones anteriores nos llevan a enfatizar la necesidad que se tiene de reconocer y valorar la contribución del trabajo doméstico al bienestar social, no sólo en el ámbito no remunerado sino también en el remunerado. En esta última instancia, hay que tener en cuenta que los grupos organizados de las llamadas empleadas del hogar mexicanas enfrentan condiciones laborales muy precarias y que hasta ahora han tenido poco eco en sus demandas de firma de contratos y un pleno acceso a la seguridad social. ${ }^{12}$

Dicho lo anterior, hay que subrayar que las estimaciones sobre las necesidades de cuidado en México apuntan a proporciones muy considerables. Las llevadas a cabo con base en las encuestas más amplias indican que en $37 \%$ de los hogares mexicanos hay un niño/a de 0 a 6 años y/o una persona adulta mayor de 60 años que requieren de cuidados (Galindo, García y Rivera, 2015). Asimismo, las estimaciones sobre la demanda futura de cuidados arrojan cifras de llamar la atención (Max, 2016; Sosa, Mejía y Soberón, 2016). Si las condiciones actuales y los volúmenes poblacionales proyectados persisten, la demanda agregada de cuidados se duplicaría para 2030 y se triplicaría para 2050 (Max, 2016). Ante este panorama, ¿cómo vemos los retos futuros más urgentes?, ¿qué estudios y prácticas deben ser estimulados?, ¿dónde y cuándo consideramos más necesaria la innovación o el mejoramiento, tanto en la investigación científica como en la política social?

Un aspecto central es la armonización u homogeneización de las fuentes de información para asegurar la calidad de la misma, la comparabilidad internacional, la valoración adecuada del trabajo no remunerado y un eventual

12 La pertinencia de estos planteamientos tiene que ser evaluada en cada contexto nacional o subnacional concreto. En el caso del conjunto de América Latina, autoras como Aguirre y Ferrari (2014) consideran que en muchas encuestas de uso del tiempo latinoamericanas, la información que se recolecta sobre trabajo doméstico es más detallada que aquella que se recolecta sobre cuidado (especialmente lo que corresponde a las personas mayores y a los discapacitados dependientes), y que es importante subsanar este desbalance en el marco del diseño de las políticas de cuidado. 
y oportuno seguimiento de programas sociales específicos. ${ }^{13}$ Sabemos que las estadísticas y los indicadores de uso del tiempo y trabajo no remunerado pueden ser afectados por aspectos tales como los tiempos de referencia, la edad de la población objeto de estudio, el marco conceptual, el tipo de cuestionario, la cobertura y el desglose de las actividades, entre otros aspectos. En nuestro caso, como hemos visto, se ha avanzado mucho hacia el levantamiento de encuestas específicas de uso del tiempo, pero todavía hay mucho que hacer en cuanto a la estandarización de los cuestionarios y la captación de las actividades simultáneas, para mencionar sólo los aspectos que despiertan las mayores controversias. Asimismo, sería deseable lograr mayor comparabilidad en los diferentes clasificadores de las actividades que se definen de un levantamiento al otro. Debido al hecho de que en las encuestas de uso del tiempo se captan tanto las tareas remuneradas como las no remuneradas, en su generación y análisis pueden converger especialistas en cuentas nacionales, censos de población y mercados de trabajo, campos en los cuales todavía prevalecen diferentes clasificadores que no siempre son modificados o actualizados de la misma manera (véase García y Pacheco, 2014a, quienes analizan algunas facetas de la Clasificación Mexicana de Actividades de Uso del Tiempo, CMAUT, en comparación con otros instrumentos de clasificación análogos).

En lo que concierne al análisis de la información sobre trabajo no remunerado en estudios de corte académico, es preciso reconocer que en pocos años se ha cubierto en México una cantidad muy relevante de temáticas. Muchas de estas investigaciones han sido promovidas tanto por ONU Mujeres como por el Instituto Nacional de las Mujeres (Inmujeres), instituciones que han destacado por su apoyo a la generación de información y a la publicación de resultados en el campo que nos ocupa. Un aspecto que merece ser subrayado es el interés que ha despertado el estudio del trabajo doméstico y de cuidados en investigadoras e investigadores jóvenes, quienes han publicado de manera autónoma o en colaboración con estudiosos de más larga trayectoria, en centros académicos de diversa índole o desde organizaciones de la sociedad civil. Hasta ahora son frecuentes los análisis de tipo más

13 México ha jugado un papel muy destacado en el contexto regional en la conformación de directrices y referentes conceptuales para armonizar las encuestas de uso del tiempo en América Latina y el Caribe. Este trabajo ha sido desarrollado en el marco del INEGI, institución que coordina el Grupo de Trabajo sobre Estadísticas de Género de la Conferencia Estadística de las Américas, de la Comisión Económica para América Latina (CEA/CEPAL). La División de Asuntos de Género de la CEPAL, Inmujeres y ONU Mujeres han colaborado de manera importante en estas labores. María Eugenia Gómez Luna ha sido una actora clave del proceso y es autora de la CAUTAL (Clasificación de Actividades de Uso del Tiempo para América Latina y el Caribe) (véase Gómez Luna, 2010, 2016). 
descriptivos y centrados en visibilizar los diferentes tipos de desigualdades (de género y socioeconómicas) involucrados en el ejercicio del trabajo no remunerado. Sin embargo, ya existe también un grupo importante de estudios, que hemos destacado antes, los cuales hacen hincapié en los factores intervinientes, en los aspectos que hay que tener en cuenta o controlar en términos estadísticos, para lograr un análisis más completo y refinado de las tendencias prevalecientes sobre trabajo doméstico y de cuidados en el país.

Por último, quisiéramos discutir los desafíos que tenemos por delante en el terreno de las políticas públicas. Ya existen importantes recuentos en el ámbito latinoamericano sobre lo avanzado por los distintos países en lo que respecta tanto a la generación de información sobre uso del tiempo y trabajo no remunerado, como a la valoración económica del mismo, y al diseño y puesta en práctica de programas y políticas específicas en este campo (véase en especial, PNUD, OIT e Inmujeres, 2009; Aguirre y Ferrari, 2014; Batthyány, 2015a). Asimismo, encontramos que en algunas investigaciones llevadas a cabo en (o para) México se aportan datos valiosos sobre la situación que prevalece en el país en torno a los servicios de cuidado que ofrece el Estado mexicano, o las (escasas) medidas existentes mediante las cuales se pudiera lograr mayor corresponsabilidad entre mujeres, hombres, Estado y comunidad, en lo que toca al trabajo y la familia (véase García y Oliveira, 2014; Galindo, García y Rivera, 2015).

Con base en esta documentación, es posible plantear que, en un contexto regional de muchas carencias, México no destaca por los programas o servicios concretos existentes que pudieran permitir aligerar o balancear la carga de trabajo remunerado que hoy recae principalmente en las mujeres. El problema sí ha sido reconocido públicamente por las instituciones gubernamentales de diversas maneras, como vimos antes en lo concerniente a la generación y el perfeccionamiento de la información estadística de acceso abierto, y a la valoración de este tipo de trabajo mediante la elaboración de las cuentas satélite de trabajo no remunerado como parte de la contabilidad nacional. Asimismo, en los planes nacionales de desarrollo y de manera más específica en el último programa que busca la igualdad de oportunidades y la no discriminación hacia las mujeres, la meta de alcanzar la corresponsabilidad en el ejercicio del trabajo no remunerado está muy presente, junto al objetivo de fomentar la presencia femenina en el trabajo remunerado y de facilitar la actividad microempresarial de las mujeres (véase el Proigualdad, 2013-2018). También habría que mencionar que en la nueva Ley Federal del Trabajo, aprobada en 2012, se reconoce la necesidad de mejorar la reconciliación trabajo-familia en el país; aunque hay que subrayar que México no ha ratificado los convenios de la Organización Internacional del Trabajo 
(OIT) referidos a los trabajadores con responsabilidades familiares, la protección a la maternidad y los trabajadores domésticos.

Más allá de los avances aquí mencionados, los análisis comparativos sobre los servicios de cuidado o la organización social del cuidado en la región latinoamericana dejan claro las insuficiencias que enfrentamos, aun frente a países de desarrollo similar al nuestro. La situación mejor conocida se refiere a los servicios de cuidado infantil, y ha sido documentado el reducido porcentaje de niños y niñas que en nuestro caso asiste a una guardería pública o privada. Las estimaciones llevadas a cabo por diferentes autores, con base en distintas fuentes, indican que solamente de 4 a $10 \%$ de niños y niñas de 0 a 5 años asiste a una guardería o centro de educación infantil, en comparación con cifras cercanas a $30 \%$ en países como Chile, Colombia y Brasil. Las madres indican que no utilizan estos servicios principalmente porque no tienen necesidad, porque no confían en ellos o porque no tienen dinero para pagarlos. No sólo se trataría del reducido número de establecimientos y de su calidad, sino de problemas como la fragmentación, la falta de coordinación y la insuficiente capacidad del Estado para inspeccionarlos y vigilarlos (véase Mateo, Rodríguez y Grafe, 2014; Galindo, García y Rivera, 2015). En este contexto habría que subrayar que la modificación del panorama descrito también tiene que pasar por un cambio sociocultural en las percepciones prevalecientes, pues en amplios sectores de la población se sigue considerando que el cuidado infantil es una responsabilidad de las mujeres y un asunto privado y familiar (véase Salazar, Salazar y Rodríguez, 2011; García y Oliveira, 2014).

A partir de lo anterior, podemos concluir que nos falta entonces mucho camino por recorrer hacia lo que sería un sistema de cuidados, entendido como un conjunto de acciones públicas y privadas intersectoriales, desarrolladas de manera articulada con el objetivo de brindar atención y apoyar a las familias en el cuidado de los miembros del hogar. Ejemplos de acciones en esta dirección en América Latina han sido documentados para los casos de Argentina, Chile, Colombia, Costa Rica, Cuba, Ecuador, y sobre todo ha recibido especial atención el diseño y puesta en práctica del Sistema Nacional de Cuidados (SNIC) en Uruguay en 2015 (Aguirre y Ferrari, 2014; Batthyány, 2015a; Ferreyra, 2016; Informe SNIC, 2016). El SNIC constituye un avance hacia la universalización de los derechos sociales en la región y en el mundo, aunque se enfrenta todavía a un presupuesto restringido. El sistema busca ampliar y crear servicios institucionales de cuidado para la primera infancia y las personas en situación de dependencia, y además contempla diferentes modalidades de atención, como serían los asistentes personales y la teleasistencia. Habría que subrayar, finalmen- 
te, que el SNIC ha surgido después de un prolongado esfuerzo de colaboración y movilizaciones por parte de distintos actores en la sociedad uruguaya, incluidos los grupos académicos. Se trata de un ejemplo del cual tenemos mucho que aprender para mejorar la organización social del trabajo no remunerado en México.

\section{Bibliografía}

Aguirre, R. y Ferrari, F. (2014). Las encuestas sobre uso del tiempo y trabajo no remunerado en América Latina y el Caribe. Caminos recorridos $y$ desafios hacia el futuro (Serie Asuntos de Género, 122). Santiago de Chile: Comisión Económica para América Latina (CEPAL). Recuperado de https://repositorio.cepal.org/bitstream/handle/11362/5851/9/ S1420397 es.pdf

Arango, L. G. y Molinier, P. (2011). El cuidado como ética y como trabajo. En L. G. Arango Gaviria y P. Molinier (coords.), El trabajo y la ética del cuidado (pp. 15-21). Bogotá, Colombia: La Carreta / Universidad Nacional de Colombia.

Arango, L. G. y Molinier, P. (2011a). El trabajo y la ética del cuidado. Bogotá, Colombia: La Carreta / Universidad Nacional de Colombia.

Arriagada, I. (2017). Familias y hogares en América Latina. En J. Nájera, B. García y E. Pacheco (coords.), Hogares y trabajadores en México en el siglo XXI, Ciudad de México: El Colegio de México, A.C.

Arriagada, I. y Todaro, R. (2012). Cadenas globales de cuidados: el papel de las migrantes peruanas en la provisión de cuidados en Chile. Santiago de Chile: ONU Mujeres.

Ariza, M. (2014). Care circulation, absence and affect in transnational families. En L. Baldassar y L. Merla (eds.), Transnational families, migration and the circulation of care. Understanding mobility and absence in family life (pp. 94-115). Nueva York: Routledge.

Batthyány, K. (ed.). (2015). Los tiempos del bienestar social. Género, trabajo no remunerado y cuidados en Uruguay. Montevideo: Inmujeres / Mides (Ministerio de Desarrollo Social).

Batthyány, K. (2015a). Las políticas y el cuidado en América Latina: una mirada a las experiencias regionales (Serie Asuntos de Género, 124). Santiago de Chile: ONU, CEPAL / Cooperación Española. Recuperado de https://repositorio.cepal.org/handle/11362/37726

Carrasco, C., Borderías, C. y Torns, T. (2011). Introducción. El trabajo de cuidados: antecedentes históricos y debates actuales. En C. Carrasco, 
C. Borderías y T. Torns (eds.), El trabajo de cuidados. Historia, teoría y politicas (pp. 13-95). Madrid, España: Catarata.

Ceballos, G. (2013). La intensidad de los trabajos de cuidados no remunerados de las mujeres en los hogares urbanos de México. Análisis con datos de la ELCOS 2012. En E. Pacheco (coord.), Los cuidados y el trabajo en México. Un análisis de la Encuesta Laboral y de Corresponsabilidad Social (ELCOS) 2012 (Cuadernos de Trabajo, 40) (pp. 125188). Ciudad de México: Instituto Nacional de las Mujeres (Inmujeres). Recuperado de http://web.inmujeres.gob.mx/transparencia/archivos/ estudios_opiniones/cuadernos/ct40.pdf

Cerrutti, M., Maguid, A. y Binstock, G. (2013). Familia, migración y cadenas globales de cuidado. En L. Pautassi y C. Zibecchi (coords.), Las fronteras del cuidado (pp. 191-214). Buenos Aires, Argentina: Biblos.

Coubes, M.-L. (2014). Entradas y salidas del mercado laboral durante el curso de vida: trayectorias laborales de tres cohortes de mujeres mexicanas. Ponencia presentada en la XII Reunión Nacional de Investigación Demográfica en México. México: Sociedad Mexicana de Demografía.

Damián, A. (2014a). La captación del uso del tiempo y la medición de la pobreza de tiempo. Algunas reflexiones sobre la experiencia en México. En B. García y E. Pacheco (coords.), Uso del tiempo y trabajo no remunerado en México (pp. 115-170). Ciudad de México: El Colegio de México, A.C. / ONU-Mujeres / Instituto Nacional de las Mujeres (Inmujeres).

Damián, A. (2014b). El tiempo, la dimensión olvidada en los estudios de pobreza y bienestar. Ciudad de México: El Colegio de México, A.C.

De Barbieri, T. (1984). Mujeres y vida cotidiana. Ciudad de México: Fondo de Cultura Económica / Universidad Nacional Autónoma de México, Instituto de Investigaciones Sociales.

Durán Heras, M. A. (2012). El trabajo no remunerado en la economía global. Bilbao, España: Fundación BBVA.

Encuesta Nacional de Uso del Tiempo (ENUT). (2014). Aguascalientes, México: Instituto Nacional de Estadística y Geografía (INEGI).

Esquivel, V. (2012a). Cuidado, economía y agendas políticas: una mirada conceptual sobre la "organización social del cuidado" en América Latina. En V. Esquivel (ed.), La economía feminista desde América Latina. Una hoja de ruta sobre los debates actuales en la región (pp. 141-189). Santo Domingo, República Dominicana: ONU-Mujeres.

Esquivel, V. (ed.). (2012b). La economía feminista desde América Latina. Una hoja de ruta sobre los debates actuales en la región. Santo Domingo, República Dominicana: ONU-Mujeres. 
Federici, S. (2013). Revolución en punto cero. Trabajo doméstico, reproducción y luchas feministas. Madrid, España: Traficantes de sueños.

Ferreyra, M. (2016). Prácticas de cuidados: algunas experiencias. En Consejo Económico y Social de la Ciudad de México, El descuido de los cuidados. Sus consecuencias en seguridad humana y desigualdad laboral (pp. 232-293). Ciudad de México: Consejo Económico y Social de la Ciudad de México.

Florez, N., Pacheco, E. y Pedrero, M. (2013). Los cuidados y la toma de decisiones. En E. Pacheco (coord.), Los cuidados y el trabajo en México. Un análisis de la Encuesta Laboral y de Corresponsabilidad Social (ELCOS) 2012 (Cuadernos de Trabajo, 40) (pp. 189-223). Ciudad de México: Instituto Nacional de las Mujeres (Inmujeres). Recuperado de http:/web.inmujeres.gob.mx/transparencia/archivos/estudios_opiniones/ cuadernos/ct40.pdf

Folbre, N. (2014). Who cares? A feminist critique of the care economy. Nueva York: Rosa Luxemburg Stiftung.

Galindo, L. M., García, G. y Rivera, P. (2015). El trabajo de cuidado en los hogares: ¿un trabajo sólo de mujeres? (Cuadernos de Trabajo, 59). Ciudad de México: Instituto Nacional de las Mujeres (Inmujeres). Recuperado de https://www.gob.mx/cms/uploads/attachment/file/47856/ ct59.pdf

Gandini, L. y Parker, S. (2013). Diario versus cuestionario: una comparación de metodologías para la medición del trabajo remunerado y no remunerado. En L. Gandini y M. Padrón Innamorato (coords.), Población y trabajo en América Latina: abordajes teórico-metodológicos y tendencias empíricas recientes (Serie Investigaciones, 14). Río de Janeiro, Brasil: ALAP (Asociación Latinoamericana de Población).

García, B. y Pacheco, E. (coords.) (2014). Uso del tiempo y trabajo no remunerado en México. Ciudad de México: El Colegio de México, A.C. / ONU-Mujeres / Instituto Nacional de las Mujeres (Inmujeres).

García, B. y Pacheco, E. (2014a). Reflexiones sobre el estudio del uso del tiempo. En B. García y E. Pacheco (coords.), Uso del tiempo y trabajo no remunerado en México (pp. 17-52). Ciudad de México: El Colegio de México, A.C. / ONU-Mujeres / Instituto Nacional de las Mujeres (Inmujeres).

García, B. y Oliveira, O. (2014). Familias, trabajo y políticas. Encuentros y desencuentros. En S. Giorguli Saucedo y V. Ugalde (coords.), Gobierno, territorio y población: las políticas públicas en la mira (pp. 195-232). Ciudad de México: El Colegio de México, A.C., Centro de Estudios Demográficos, Urbanos y Ambientales. 
Gobierno de la República (2013). Programa Nacional para la Igualdad de Oportunidades y no Discriminación contra las mujeres, 2013-2018, Plan Nacional de Desarrollo, 2013-2018. Ciudad de México.

Goldsmith, M. (2007). De sirvientas a empleadas del hogar. La cara cambiante del servicio doméstico en México. En M. Lamas (coord.), Miradas feministas sobre los mexicanos del siglo XX (pp. 279-311). Ciudad de México: Fondo de Cultura Económica.

Gómez Luna, M. E. (2010). Directrices y referentes conceptuales para armonizar las encuestas de uso del tiempo en América latina y el Caribe. Ciudad de México: CEA - CEPAL / INEGI / Inmujeres / Unifem.

Gómez Luna, M. E. (2016). Clasificación de Actividades de Uso del Tiempo para América Latina y el Caribe (CAUTAL). Santiago de Chile: Conferencia Estadística de las Américas de la Comisión Económica para América Latina (CEPAL) / ONU Mujeres / Instituto Nacional de Estadística y Geografía (INEGI) / Instituto Nacional de las Mujeres (Inmujeres).

Granados, A. (2013). Participación de los hombres en el cuidado no remunerado en las ciudades de México, Guadalajara y Monterrey y las zonas urbanas de México. En E. Pacheco (coord.), Los cuidados y el trabajo en México. Un análisis a partir de la Encuesta Laboral y de Corresponsabilidad Social (ELCOS) 2012 (Cuadernos de Trabajo, 40) (pp. 279301). Ciudad de México: Instituto Nacional de las Mujeres (Inmujeres). Recuperado de http://web.inmujeres.gob.mx/transparencia/archivos/ estudios_opiniones/cuadernos/ct40.pdf

Hernández, A. (2013). ¿Cuidar y trabajar para el mercado? Expectativas laborales de las mujeres cuidadoras no económicamente activas en México, 2012. En E. Pacheco (coord.), Los cuidados y el trabajo en México. Un análisis a partir de la Encuesta Laboral y de Corresponsabilidad Social (ELCOS) 2012 (Cuadernos de Trabajo, 40) pp. 224- 248). Ciudad de México: Instituto Nacional de las Mujeres (Inmujeres). Recuperado de http://web.inmujeres.gob.mx/transparencia/archivos/estudios_ opiniones/ cuadernos/ct40.pdf

Informe Anual del Sistema de Cuidados. (2016). Montevideo, Uruguay: Sistema Nacional Integrado de Cuidados. Recuperado de http://www. sistemadecuidados.gub.uy/innovaportal/file/84284/1/informe-anual2016-snic-1.pdf.

Instituto Nacional de las Mujeres (Inmujeres). (2005). Pobreza, género y uso del tiempo. Ciudad de México: Instituto Nacional de las Mujeres (trabajo elaborado por Silvia Luna). Recuperado de http://cedoc.inmujeres. gob.mx/documentos_download/100719.pdf 
Jácome, T. (2013). ¿Quién cuida a quién? Diferencias entre mujeres y hombres que realizan trabajo de cuidado en el hogar. En E. Pacheco (coord.), Los cuidados y el trabajo en México. Un análisis a partir de la Encuesta Laboral y de Corresponsabilidad Social (ELCOS) 2012 (Cuadernos de Trabajo, 40) (pp. 32-69). Ciudad de México: Instituto Nacional de las Mujeres (Inmujeres). Recuperado de http://web.inmujeres.gob.mx/ transparencia/archivos/estudios_opiniones/cuadernos/ct40.pdf

Jácome, T. Mier y Terán, M. (2014). El uso del tiempo entre los miembros de hogares indígenas y no indígenas. En B. García y E. Pacheco (coords.), Uso del tiempo y trabajo no remunerado en México (pp. 325-379). Ciudad de México: El Colegio de México, A.C. / ONU-Mujeres / Instituto Nacional de las Mujeres (Inmujeres).

López Ortega, M., Licea, R. M., Figueroa, R. y García, A. (2016). Valor económico de los cuidados a personas de 60 años y más en México. Estimación y retos para su provisión. Ponencia presentada en la XIII Reunión Nacional de Investigación Demográfica en México, Sociedad Mexicana de Demografía (Somede), Instituto Nacional de Geriatría e Instituto Nacional de Estadística y Geografía (INEGI), 22 a 24 de junio.

Márquez, C. (2017). Buscando la conciliación. El papel del trabajo remunerado y el no remunerado en los tránsitos hacia la exclusión laboral de las mujeres mexicanas urbanas. En J. Nájera, B. García y E. Pacheco (coords.), Hogares y trabajadores en México en el siglo XXI (pp. 327364). Ciudad de México: El Colegio de México, A.C., Centro de Estudios Demográficos, Urbanos y Ambientales.

Márquez, C. y Mora, M. (2014). Inequidades de género y patrones de uso del tiempo: exploración a partir del desempleo encubierto. En B. García y E. Pacheco (coords.), Uso del tiempo y trabajo no remunerado en México (pp. 509-569). Ciudad de México: El Colegio de México, A.C. / ONU-Mujeres / Instituto Nacional de las Mujeres (Inmujeres).

Martín, M. T. (2011). "Domesticar" el trabajo: una reflexión a partir de los cuidados. En L. G. Arango Gaviria y P. Molinier (coords.), El trabajo y la ética del cuidado (pp. 67-89). Bogotá, Colombia: La Carreta / Universidad Nacional de Colombia.

Martínez, J. y Voorend, K. (2013). Desigualdades de género en los regímenes de bienestar latinoamericanos: mercado, política social y organización familiar de los cuidados. En L. Pautassi y C. Zibecchi (coords.), Las fronteras del cuidado. Agenda, derechos e infraestructura (pp. 59-97). Tucumán, Argentina: Biblos.

Mateo, M., Rodríguez, L. y Grafe, F. (2014). Ley de guarderías en México 
y los desafios institucionales de conectar familia y trabajo. Resumen de políticas. Ciudad de México: Banco Interamericano de Desarrollo (BID). Max Monroy, E. E. (2016). Demanda de cuidados de los adultos mayores: situación actual y escenarios a futuro. Tesis de maestría en Demografía, El Colegio de México, A.C., Centro de Estudios Demográficos, Urbanos y Ambientales.

Miranda, S. (2013). Niñas que cuidan niños. El aporte de las niñas y las adolescentes a las actividades de cuidados en los hogares de las zonas urbanas de México. En E. Pacheco (coord.), Los cuidados y el trabajo en México. Un análisis a partir de la Encuesta Laboral y de Corresponsabilidad Social (ELCOS) 2012 (Cuadernos de Trabajo, 40) (pp. 249279). Ciudad de México: Instituto Nacional de las Mujeres (Inmujeres). Recuperado de http://web.inmujeres.gob.mx/transparencia/archivos/ estudios_opiniones/cuadernos/ct40.pdf

Montaño, S. y Calderón, C. (coords.). (2010). El cuidado en acción: entre el derecho y el trabajo (Cuadernos de la CEPAL, 94). Santiago de Chile: CEPAL. Recuperado de https://repositorio.cepal.org/bitstream/ handle/11362/27845/1/S2010994_es.pdf

Nava, I. (2013). Actividades de cuidado, mercado de trabajo remunerado y ciclo de vida familiar en las mujeres urbanas de México. En E. Pacheco (coord.), Los cuidados y el trabajo en México. Un análisis a partir de la Encuesta Laboral y de Corresponsabilidad Social (ELCOS) 2012 (Cuadernos de Trabajo, 40) (pp. 70-102). Ciudad de México: Instituto Nacional de las Mujeres (Inmujeres). Recuperado de http://web.inmu jeres.gob.mx/transparencia/archivos/estudios_opiniones/cuadernos/ ct40 pdf

Negrete Prieto, R. y Ramírez Reynoso, T. (2015). Resolución I de la XIX Conferencia Internacional de Estadísticos del Trabajo y su impacto en la estadística laboral en México. Realidad, Datos y Espacio. Revista Internacional de Estadística y Geografia, 6(1), 92-131. Recuperado de https:// www.inegi.org.mx/rde/2015/01/04/resolucion-i-de-la-xix-conferenciainternacional-de-estadisticos-del-trabajo-y-su-impacto-en-la-estadisticalaboral-en-mexico/

Oliveira, O. Ariza, M. y Eternod, M. (1996). Trabajo e inequidad de género. En O. de Oliveira, M. Ariza, M. Eternod, M. López y V. Salles (coords.), Informe final. La condición femenina: una propuesta de indicadores. Ciudad de México: Sociedad Mexicana de Demografía (Somede) / Consejo Nacional de Población (no publicado).

Organización Internacional del Trabajo (OIT). (2013). Resolución 1. Resolución sobre las estadísticas del trabajo, la ocupación y la subutilización 
de la fuerza de trabajo. Ginebra. Recuperado de: http://ilo.org/global/ statistics-and-databases/meetings-and-events/international-conferenceof-labour-statisticians/19/WCMS_234036/lang--es/index.htm

Orozco, K. (2013). Las tareas del cuidado: reflejo de una barrera y diferenciada inserción laboral. En E. Pacheco (coord.), Los cuidados y el trabajo en México. Un análisis a partir de la Encuesta Laboral y de Corresponsabilidad Social (ELCOS) 2012 (Cuadernos de Trabajo, 40) (pp. 103-124). Ciudad de México: Instituto Nacional de las Mujeres (Inmujeres). Recuperado de http://web.inmujeres.gob.mx/transparencia/archivos/ estudios_opiniones/cuadernos/ct40.pdf

Orozco, K. (2014). El papel de las cargas domésticas y los arreglos familiares en el trabajo asalariado urbano de México, 2009. Tesis de doctorado en Estudios de Población, El Colegio de México, A.C., Centro de Estudios Demográficos, Urbanos y Ambientales.

Orozco, K. (2017). Reparto del trabajo doméstico y el extradoméstico al interior de los arreglos familiares urbanos. En J. Nájera, B. García y E. Pacheco (coords.), Hogares y trabajadores en México en el siglo XXI (pp. 277-326). Ciudad de México: El Colegio de México, A.C., Centro de Estudios Demográficos, Urbanos y Ambientales.

Pacheco, E. (2010). Arreglos familiares y división del trabajo en el hogar: familias con doble proveedor. En A. M. Chávez Galindo y C. Menkes Bancet (eds.), Procesos y tendencias poblacionales en el México contemporáneo. Una mirada desde la Enadid 2006. Ciudad de México: Universidad Nacional Autónoma de México, Centro Regional de Investigaciones Multidisciplinarias / Secretaría de Salud.

Pacheco, E. (coord.) (2013). Los cuidados y el trabajo en México. Un análisis a partir de la Encuesta Laboral y de Corresponsabilidad Social (ELCOS) 2012 (Cuadernos de Trabajo, 40). Ciudad de México: Instituto Nacional de las Mujeres (Inmujeres). Recuperado de http://web. inmujeres.gob.mx/transparencia/archivos/estudios_opiniones/cuadernos/ ct40.pdf

Pacheco, E. y Florez, N. (2014). Entre lo rural y urbano: tiempo y desigualdades de género. En B. García y E. Pacheco (coords.), Uso del tiempo y trabajo no remunerado en México (pp. 263-323). Ciudad de México: El Colegio de México, A.C. / ONU-Mujeres / Instituto Nacional de las Mujeres (Inmujeres).

Pautassi, L. y Zibecchi, C. (coords.). (2013). Las fronteras del cuidado. Agenda, derechos e infraestructura. Tucumán, Argentina: Biblos.

Pedrero, M. (1977). La participación femenina en la actividad económica y su presupuesto de tiempo. Notas sobre problemas relativos a conceptos 
y captación (Avances de Investigación, 3). Ciudad de México: Centro Nacional de Información y Estadísticas del Trabajo / Secretaría del Trabajo y Previsión Social.

Pedrero, M. (2014). Importancia del trabajo no remunerado: su medición y valoración por medio de las encuestas de uso del tiempo. En B. García y E. Pacheco (coords.), Uso del tiempo y trabajo no remunerado en México (pp. 53-114). Ciudad de México: El Colegio de México, A.C. / ONU-Mujeres / Instituto Nacional de las Mujeres (Inmujeres).

PNUD, OIT e Inmujeres (2009). Trabajo y familia: hacia nuevas formas de conciliación con corresponsabilidad social. Santiago de Chile: Programa de las Naciones Unidas para el Desarrollo (PNUD) / Organización Internacional del Trabajo (OIT) / Instituto Nacional de las Mujeres de México.

Proigualdad. (2013). Programa Nacional para la Igualdad de Oportunidades y No Discriminación contra las Mujeres, 2013-2018. Diario Oficial de la Federación, 719(22). Ciudad de México: Gobierno de la República. Recuperado de https://www.gob.mx/cms/uploads/attachment/file/11895/ Programa-Nacional-para-la-Igualdad.pdf

Rendón, T. (2008). Trabajo de hombres y trabajo de mujeres en el México del siglo XX. Ciudad de México: Universidad Nacional Autónoma de México.

Rivero, E. y Hernández, A. (2014). No todo el tiempo es igual: variaciones en los patrones de uso del tiempo en México. En B. García y E. Pacheco (coords.), Uso del tiempo y trabajo no remunerado en México (pp. 221-262). Ciudad de México: El Colegio de México, A.C. / ONUMujeres / Instituto Nacional de las Mujeres (Inmujeres).

Rodríguez, C. (2012). La cuestión del cuidado: ¿el eslabón perdido del análisis económico? Revista CEPAL, 106, 23-36. Recuperado de https:// repositorio.cepal.org/bitstream/handle/11362/11524/106023036_es.pdf

Rodríguez, D. y Cooper, J. (comps.). (2005). El debate sobre el trabajo doméstico. Ciudad de México: Universidad Nacional Autónoma de México (UNAM), Instituto de Investigaciones Económicas, Escuela Nacional de Trabajo Social, Centro de Investigaciones Interdisciplinarias en Ciencias y Humanidades.

Rodríguez, M. y García, B. (2014). Trabajo doméstico y de cuidado masculino. En B. García y E. Pacheco (coords.), Uso del tiempo y trabajo no remunerado en México (pp. 381-431). Ciudad de México: El Colegio de México, A.C. / ONU-Mujeres / Instituto Nacional de las Mujeres (Inmujeres).

Rodríguez, M. y García, B. (2017). Trabajo no remunerado en México: un 
análisis de las diferencias de género en los estados y municipios del país. Ciudad de México: El Colegio de México, A.C., Centro de Estudios Demográficos, Urbanos y Ambientales.

Rojas, O. y Martínez, M. (2014). Uso del tiempo en el ámbito doméstico entre los padres mexicanos. En B. García y E. Pacheco (coords.), Uso del tiempo y trabajo no remunerado en México (pp. 433-469). México: El Colegio de México, A.C. / ONU-Mujeres / Instituto Nacional de las Mujeres (Inmujeres).

Salazar, R., Salazar, H. y Rodríguez, M. (coords.). (2011). Conciliación, trabajo y familia en México: las responsabilidades compartidas de mujeres y hombres en el debate público (Análisis Político). Ciudad de México: Friedrich Ebert Stiftung.

Sánchez, L. (2014). El trabajo doméstico en las parejas de doble ingreso en México. En B. García y E. Pacheco (coords.), Uso del tiempo y trabajo no remunerado en México (pp. 471-507). Ciudad de México: El Colegio de México, A.C. / ONU-Mujeres / Instituto Nacional de las Mujeres (Inmujeres).

Sánchez, M. J. y Martini, M. F. (1987). Trabajo doméstico y reproducción social. Un estudio de caso en la colonia Santa Úrsula Xitla. Tesis de maestría en Antropología Social. México: Escuela Nacional de Antropología e Historia (ENAH).

Santoyo, L. y Pacheco, E. (2014). El uso del tiempo de las personas en México según tipo de hogar. Una expresión de las desigualdades de género. En B. García y E. Pacheco (coords.), Uso del tiempo y trabajo no remunerado en México (pp. 171-219). Ciudad de México: El Colegio de México, A.C. / ONU-Mujeres / Instituto Nacional de las Mujeres (Inmujeres).

Sosa, M. V., Mejía, A. y Soberón, J. A. (2016). Desaceleración del crecimiento poblacional: impacto a mediano plazo en el cuidado de personas en México a inicios del siglo XXI. Ponencia presentada en la XIII Reunión Nacional de Investigación Demográfica en México, Sociedad Mexicana de Demografía (Somede), 22 a 24 de junio.

Torns, T. (2008). El trabajo y el cuidado: cuestiones teórico-metodológicas desde la perspectiva de género. Empiria. Revista de Metodología de Ciencias Sociales, 15, 53-73. Recuperado de http://revistas.uned.es/ index.php/empiria/article/view/1199/1102

Torres, C. (1989). El trabajo doméstico y las amas de casa. El rostro invisible de las mujeres. Sociológica. Revista del Departamento de Sociología, 4(10), 145-176. Recuperado de http://www.sociologicamexico.azc.uam. mx/index.php/Sociologica/article/view/985/957 
Vega, C. y Gutiérrez, E. (2014). Nuevas aproximaciones a la organización social del cuidado. Debates Latinoamericanos. Presentación del dossier. Íconos, Revista de Ciencias Sociales, 50, 9-26. Recuperado de http:// www.redalyc.org/articulo.oa?id $=50931716001$

Yeates, N. (2005). A global political economy of care. Social Policy and Society, 4(2), 227-234. Recuperado de https://www.cambridge.org/core/ services/aop-cambridge-core/content/view/97DECD989A6955E2654F 5322F742377C/S1474746404002350a.pdf/global_political_economy_ of_care.pdf

\section{Acerca de la autora}

Brígida García es profesora-investigadora de El Colegio de México, A.C. Es maestra en Demografía por esta misma institución y doctora en Sociología por la Universidad Nacional Autónoma de México. Ha publicado diversos libros, más de un centenar de artículos en revistas especializadas y capítulos en libros en los temas de familias, trabajo y género. Ha sido Presidenta de la Sociedad Mexicana de Demografía (Somede) y es Investigadora Emérita del Sistema Nacional de Investigadores. Sus publicaciones recientes se han concentrado en los temas de hogares y trabajadores, uso del tiempo y trabajo no remunerado. ORCID: https://orcid.org/0000-0003-2223-5225

Su libro más reciente es:

Nájera, J., García, B. y Pacheco, E. (coords.) (2017). Hogares y trabajadores en el México del siglo XXI. Ciudad de México: El Colegio de México, A.C.

Recepción: 20 de octubre de 2017.

Aceptación: 13 de abril de 2018. 
